\title{
Improving the active expression of transglutaminase in Streptomyces lividans by promoter engineering and codon optimization
}

\author{
Song Liu ${ }^{1 *}$, Miao Wang ${ }^{4}$, Guocheng Du ${ }^{1,3^{*}}$ and Jian Chen ${ }^{1,2}$
}

\begin{abstract}
Background: Transglutaminases (TGase), which are synthesized as a zymogen (pro-TGase) in Streptomyces sp., are important enzymes in the food industry. Because this pro-peptide is essential for the correct folding of Streptomyces TGase, TGase is usually expressed in an inactive pro-TGase form, which is then converted to active TGase by the addition of activating proteases in vitro. In this study, Streptomyces hygroscopicus TGase was actively produced by Streptomyces lividans through promoter engineering and codon optimization.

Results: A gene fragment (tg1, $2.6 \mathrm{~kb})$ that encoded the pro-TGase and its endogenous promoter region, signal peptide and terminator was amplified from S. hygroscopicus WSH03-13 and cloned into plasmid plJ86, which resulted in plJ86/tg1. After fermentation for 2 days, S. lividans TK24 that harbored plJ86/tg1 produced 1.8 U/mL of TGase, and a clear TGase band (38 kDa) was detected in the culture supernatant. These results indicated that the pro-TGase was successfully expressed and correctly processed into active TGase in S. lividans TK24 by using the TGase promoter. Based on deletion analysis, the complete sequence of the TGase promoter is restricted to the region from -693 to -48 . We also identified a negative element $(-198$ to -148$)$ in the TGase promoter, and the deletion of this element increased the TGase production by $81.3 \%$, in contrast to the method by which S. lividans expresses plJ86/tg1. Combining the deletion of the negative element of the promoter and optimization of the gene codons, the yield and productivity of TGase reached $5.73 \mathrm{U} / \mathrm{mL}$ and $0.14 \mathrm{U} / \mathrm{mL} / \mathrm{h}$ in the recombinant $S$. lividans, respectively.
\end{abstract}

Conclusions: We constructed an active TGase-producing strain that had a high yield and productivity, and the optimized TGase promoter could be a good candidate promoter for the expression of other proteins in Streptomyces.

Keywords: Transglutaminase, Endogenous promoter, Codon optimization, Streptomyces hygroscopicus, Streptomyces lividans

\section{Background}

Transglutaminase (TGase, EC 2.3.2.13) is an enzyme that exhibits several catalytic activities: the crosslinking of proteins by forming $\mathrm{N}^{\varepsilon}$ - $(\gamma$-glutamyl) lysine bonds, the incorporation of polyamines into protein, and the deamidation of protein-bound glutamines [1]. Because of

\footnotetext{
* Correspondence: liusong@jiangnan.edu.cn; gcdu@jiangnan.edu.cn 'Key Laboratory of Industrial Biotechnology, Ministry of Education, School of Biotechnology, Jiangnan University, Wuxi, China

Full list of author information is available at the end of the article
}

these catalytic abilities, TGase has been widely used in industrial processing, especially in food processing, for improving the functional properties of various proteins, including meat, soy, myosin, globulin, casein, peanut, and whey proteins [2]. TGase is widely distributed in various organisms, including plants [3], mammals [4], and microorganisms [5]. Among the TGases, the TGase from Streptomyces is $\mathrm{Ca}^{2+}$-independent and is advantageous for industrial applications because it has a higher reaction rate, broad substrate specificity for an acyl 
donor, and a smaller molecular size [6, 7]. The development of an efficient and easy-to-use expression system for the production of Streptomyces TGase is therefore highly desirable.

Streptomyces TGase is secreted as pro-TGase and becomes active after the cleavage of the pro-peptide by endogenous activating proteases [5]. Because the propeptide is essential for the correct folding of TGase, direct expression of mature TGase yields insoluble inclusion bodies [8] or inactive enzyme [9]. Thus, TGase is usually expressed in a pro-TGase form $[10,11]$. Due to the absence of activating protease in the host strain, coexpression of heterologous proteases is required to convert pro-TGase into active TGase [12]. Because of the ability to convert the pro-protein into the active enzyme with its own proteases, Streptomyces hosts became ideal hosts for producing active TGase. TGases from Streptoverticillium mobaraense, Streptoverticillium ladakanum, and Streptomyces platensis have been heterologously expressed in Streptomyces lividans as an active enzyme [13-15]. However, the secretion level of TGase in $S$. lividans 3131 is less than $0.01 \mathrm{U} / \mathrm{mL}$ [13]. When $S$. lividans JT46 was used as the host strain, the yield of TGase reaches only $1.23-2.22 \mathrm{U} / \mathrm{mL}$ after 3-6 days of fermentation $[14,15]$. Overall, both the yield and productivity of TGase as expressed in Streptomyces hosts are still low.

The ermE and tipA promoters have proven to be highly successful for the over-expression of Streptomyces genes [16]. However, the ermE promoter improved TGase production by $0.8 \mathrm{U} / \mathrm{mL}$ [17], and there are no reports for TGase expression with the other strong promoters. It has been found that the endogenous promoter of TGase is recognized in S. lividans, and the yield of the recombinant S. platensis TGase reached $2.22 \mathrm{U} / \mathrm{mL}$ [15], which suggests that the endogenous promoter of different TGases or its modified versions could be more efficient for TGase expression by S. lividans in contrast to heterologous strong promoters. In addition, the Streptomyces genome has a high (>70 \%) GC content, and rare codons such as TTA could significantly reduce the protein expression in S. lividans [18]. However, the Streptomyces TGase gene contains rare codons such as TTA, although it was found in Streptomyces [14, 15, 19]. Thus, codon optimization could also benefit TGase expression in S. lividans.

Previously, we cloned the DNA fragment (GenBank No: HM231108) that contained the TGase gene with a flanking region sequence from the $S$. hygroscopicus genome, and a putative promoter region was found upstream to TGase [10]. In this study, the S. hygroscopicus TGase gene was expressed in S. lividans TK24 by using its putative endogenous promoter. Then, the putative promoter was partially deleted, and the effects of the deletions on the expression of TGase in S. lividans TK24 were analyzed. In addition, the codons of TGase were optimized to further enhance the level of TGase expression. Finally, a relatively high level of TGase expression in S. lividans was achieved.

\section{Results \\ Expression of TGase in S. lividans using its endogenous promoter}

To express the TGase in S. lividans using its endogenous promoter, a gene fragment (tg1, $2.6 \mathrm{~kb})$ was amplified from the S. hygroscopicus genome (Fig. 1a) and cloned into pIJ86, which resulted in the plasmid pIJ86/tg1 (Fig. 1b). The tg 1 encoded the TGase ORF (1257 bp), the upstream sequence (893 bp) and the downstream sequence (458 bp) (Fig. 1a). As analyzed previously, the $S$. hygroscopicus TGase ORF was composed of a secretory signal peptide gene, a pro-peptide gene, and the mature TGase gene; the upstream and downstream sequence of the ORF contain a putative promoter and a putative terminator, respectively [10]. The expression vector was transformed into S. lividans TK24, yielding S. lividans TK24/pIJ86/tg1.

When cultivated for $48 \mathrm{~h}$, S. lividans TK24/pIJ86/tg1 obtained $1.8 \mathrm{U} / \mathrm{mL}$ of extracellular TGase, which was approximately 1.5 -fold of that achieved in the wild strain S. hygroscopicus WSH03-13 under the same cultivation conditions (Fig. 1c). TGase activity was not detected in the culture supernatants of the control strains S. lividans TK24/pIJ86 (S. lividans TK24 carrying pIJ86) and $S$. lividans TK24 (Fig. 1c). After treatment with TGaseactivating protease dispase [10], the culture supernatants of the control strains still did not exhibit TGase activity (data not shown). Then, the culture supernatants of $S$. lividans TK24/pIJ86/tg1, S. hygroscopicus, and the control strains were subjected to SDS-PAGE analysis. As shown in Fig. 1d (lane 1), the S. lividans TK24/pIJ86/tg1 showed a remarkable band that had a size of $38 \mathrm{kDa}$, which corresponds to the molecular weight of $S$. hygroscopicus TGase [20]. In the case of control strains, a small number of TGase/pro-TGase-like bands was detected in the culture supernatants (Fig. 1d, lanes 2 and 3). For failing to detect TGase activity in the control samples (Fig. 1c), these TGase/pro-TGase-like bands could correspond to the endogenous extracellular proteins of S. lividans TK24. Two proteins with approximate molecular weights of pro-TGase and TGase were detected in the culture supernatants of $S$. hygroscopicus (Fig. 1d, lane 4), which indicates that pro-TGase is not fully processed [10]. Because the ermE promoter was removed in $\mathrm{pIJ} 86 / \operatorname{tg} 1$, our results indicated that the upstream sequence $(893 \mathrm{bp})$ contains the endogenous promoter, which could drive the expression of TGase in S. lividans TK24. Moreover, the pro-TGase is correctly 


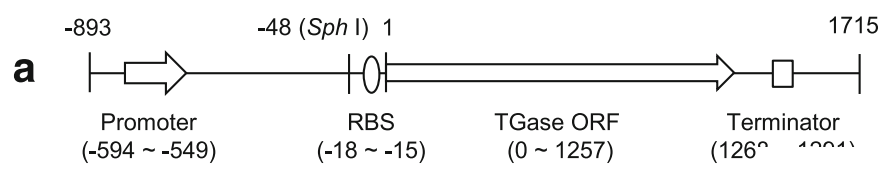

$\operatorname{tg} 1(2.6 \mathrm{~kb})$

b

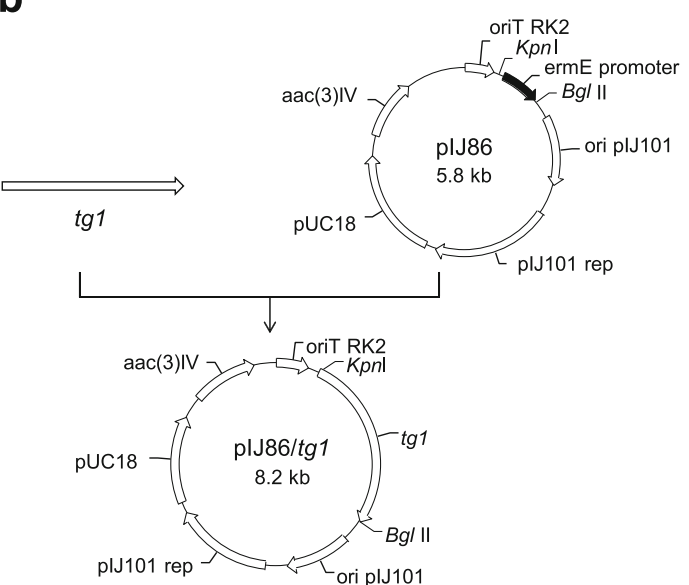

C
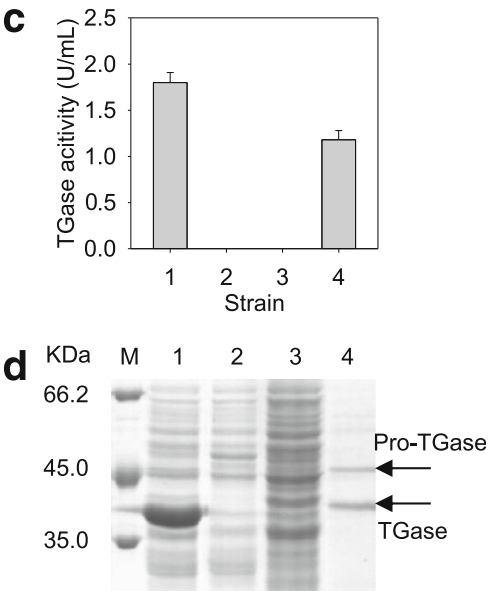

Fig. 1 Production of TGase by S. lividans TK24/pIJ86/tgl. a The gene structure of tg1. b Construction of TGase expression plasmid plJ86/tg1. c TGase activity assay of the culture supernatants of Streptomyces strains. $\mathbf{d}$ SDS-PAGE analysis of the TGases in the culture supernatants of Streptomyces strains. Labeling for (a): The numbers in the illustration indicate the base positions. Labeling for (c): 1: S. lividans TK24/plJ86/tgl (the recombinant strain that expresses the S. hygroscopicus TGase ORF using the TGase endogenous promoter), 2: S. lividans TK24/plJ86 (the control strain that carries plJ86), 3: S. lividans TK24 (the control strain without the expression plasmid), 4: S. hygroscopicus WSH03-13 (the wild type strain that produces TGase). Labels for (d): M: protein marker, 1: S. lividans TK24/plJ86/tgl, 2: S. lividans TK24/plJ86, 3: S. lividans TK24, 4: S. hygroscopicus WSH03-13. The recombinant S. lividans TK24 were inoculated into $30 \mathrm{~mL}$ of medium (which contained $50 \mathrm{\mu g} / \mathrm{mL}$ apramycine) and cultured at $30{ }^{\circ} \mathrm{C}$ and $200 \mathrm{rpm}$ for $48 \mathrm{~h}$

processed by the host proteases, which suggests that $S$. lividans TK24 is an ideal host for the active expression of TGase.

\section{Deletion analysis of the TGase promoter}

As shown in Fig. 1a, the putative core promoter was located in the upstream sequence (between $-594 \mathrm{bp}$ and $-549 \mathrm{bp}$ ) of the TGase ORF. To identify the TGase promoter, we analyzed the effect of the upstream sequence deletions on the expression of TGase in S. lividans TK24.

First, deletions at the $5^{\prime}$-end of the upstream sequence were conducted. Deleting the upstream down to -793 (pTGU2) or -693 (pTGU3) had no significant effect on the expression level of the TGase gene (Fig. 2a). However, deleting the upstream down to -593 (pTGU4) resulted in a significant decrease in the TGase production, approximately $57.6 \%$ of the activity of non-deletion (Fig. 2a). Deleting down to -493 (pTGU5) resulted in the complete loss of the TGase activity (Fig. 2a). These results suggest that the region from -693 to -493 contains important components of the TGase promoter.

Second, deletions at the 3 '-end of the upstream sequence were conducted. Because the putative ribosomebinding site was located in the upstream sequence between -18 and -15 , the 3 -end deletion was initiated at -48 . Deleting upstream from -48 up to -98 (pTGD2) did not have a significant effect on the expression level of the TGase gene (Fig. 2b). Deletion of up to -148 (pTGD2) resulted in a decrease in the TGase activity, approximately $82.1 \%$ of the activity of the non-deletion (pIJ86/tg1) (Fig. 2b). Interestingly, deleting up to -198 (pTGD4) increased the TGase activity by $27.3 \%$ (Fig. 2b). However, the deletion mutant pTGD5 resulted in a decrease in the TGase activity (91.2 \%), and further deletion (pTGD6-pTGD10) caused a significant decrease in the TGase activity (less than $40 \%$ of the activity of pIJ86/tg1) (Fig. 2b). Last, TGase activity could not be detected with the deletion mutant pTGD10 (-498) (Fig. 2b). These results suggest that the region from -498 to -198 and the region from -148 to -98 could be the positive elements for the TGase promoter, while the region from -198 to -148 was the negative element for the TGase promoter.

Based on the deletion analysis (Fig. 2), the complete promoter of TGase could be restricted to the sequence from -693 to -48 in $\mathrm{tg} 1$. Because the region from -198 to -148 negatively affected the expression, this region was deleted from the complete promoter $(-693$ to -48$)$, which yielded the TGase expression plasmid pTGO. As 
a

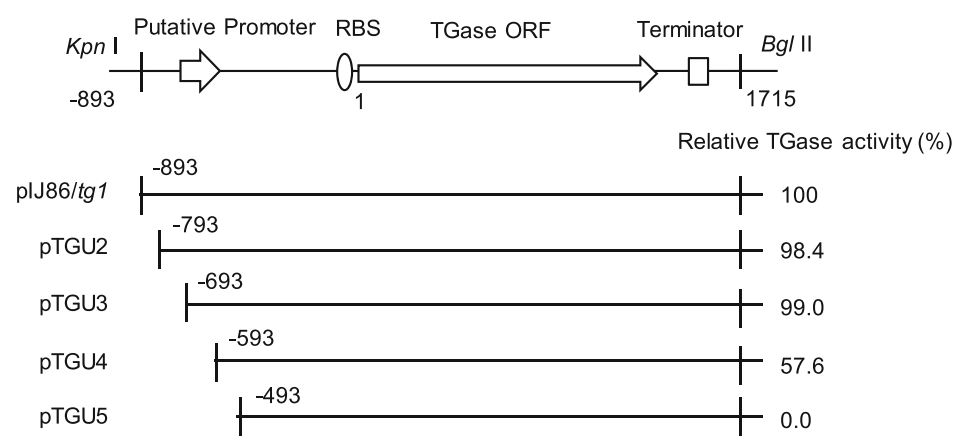

b

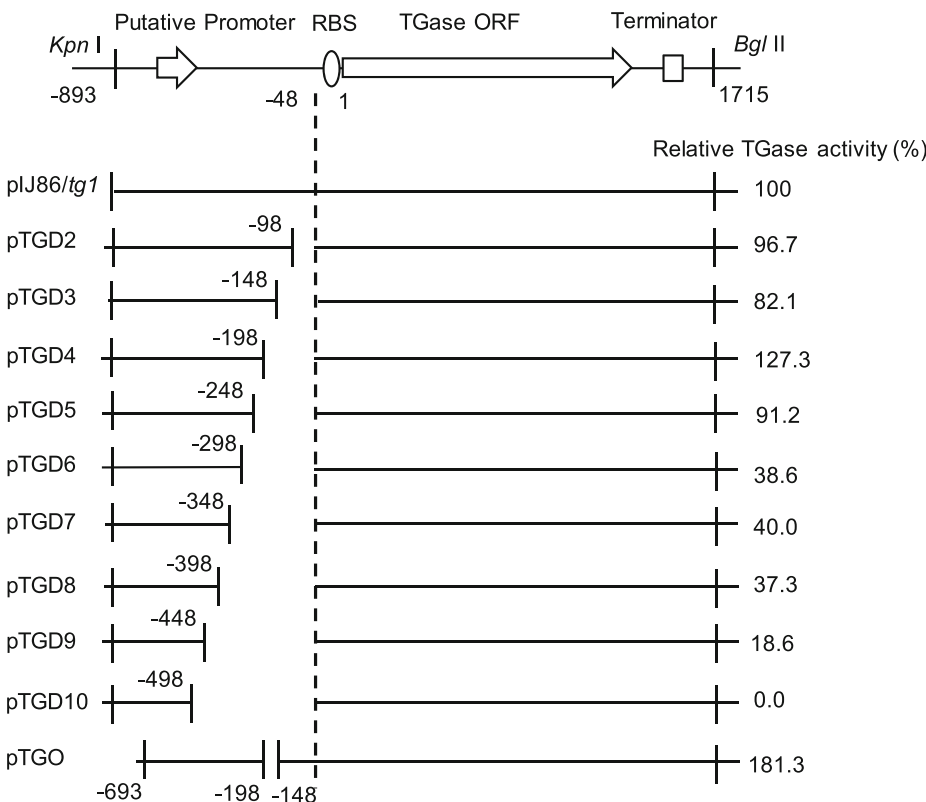

Fig. 2 The effect of the endogenous promoter modification on the expression of TGase in S. lividans. a Partially deleting the $5^{\prime}$-end of the TGase promoter region. $\mathbf{b}$ Partially deleting the $3^{\prime}$-end of the TGase promoter region. The recombinant S. lividans TK24 were inoculated into $30 \mathrm{~mL}$ of medium (which contained $50 \mu \mathrm{g} / \mathrm{mL}$ apramycine) and cultured at $30^{\circ} \mathrm{C}$ and $200 \mathrm{rpm}$ for $48 \mathrm{~h}$

indicated by Fig. 2b, cells that carried pTGO achieved $3.3 \mathrm{U} / \mathrm{mL}$ of TGase, which was $81.3 \%$ higher than that obtained by pIJ86/tg1 (Fig. 2b).

\section{Codon optimization of the TGase gene in S. lividans}

To improve the TGase expression in S. lividans, the gene sequence of TGase ORF was optimized according to the gene codon bias of Streptomyces and was chemically synthesized (Fig. 3). The codon-optimized TGase ORF along with the intact upstream $(-48$ to -1$)$ and downstream (1258 to 1715) was then cloned into the Sph I-Bgl II sites of pTGO, which yielded pTGOm. As shown in Fig. 4, when S. lividans expressed pTGOm, the highest yield of TGase $(5.73 \mathrm{U} / \mathrm{mL})$ was obtained, which was $73.6 \%$ higher than that produced by S. lividans when it harbored pTGO. Moreover, the former recombinant strain achieved the highest yield of TGase at $42 \mathrm{~h}$, while for the latter strain, the highest yield was obtained at $48 \mathrm{~h}$. Consequently, the productivity of $S$. lividans when it expressed pTGOm was $0.14 \mathrm{U} / \mathrm{mL} / \mathrm{h}$, which was twofold higher than that of S. lividans while it harbored pTGO.

\section{Discussion}

Although TGase from Sv. ladakanum [14] and S. platensis [15] has been expressed in S. lividans JT46 by TGase promoters, the yields of TGase reach only 1.23-2.22 U/ $\mathrm{mL}$ after 3-6 days of fermentation, and the productivities are less than $0.03 \mathrm{U} / \mathrm{mL} / \mathrm{h}[14,15]$. Recently, another recombinant S. lividans has obtained only $0.07 \mathrm{U} /$ $\mathrm{mL} / \mathrm{h}$ of TGase productivity by using the Streptomyces cinnamoneus phospholipase D promoter and signal 


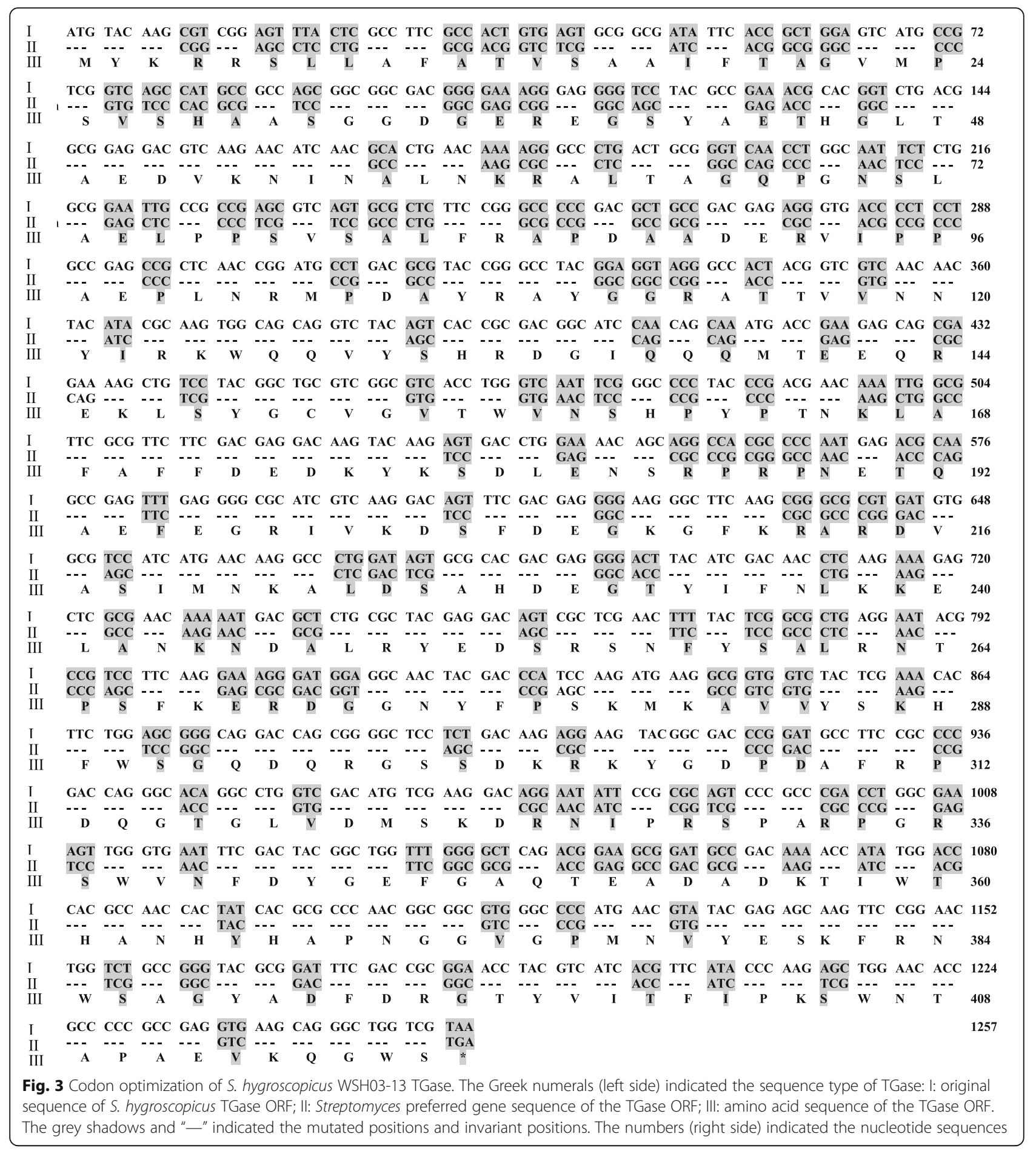

peptide [21]. In this study, S. lividans TK24 that harbored pTGM obviously obtained a higher yield (5.73 U/ $\mathrm{mL})$ and productivity $(0.14 \mathrm{U} / \mathrm{mL} / \mathrm{h})$ for the TGase (Fig. 4). It has been reported that different proteases showed variant activation efficiencies against Streptomyces pro-TGase in vitro [22]. Because all of these TGases are expressed in pro-TGase form, S. lividans TK24 could have those proteases that are more favorable for the proTGase activation in contrast to S. lividans JT46 [23].

To improve the production of TGase, the endogenous promoter of S. hygroscopicus TGase was engineered. Previously, we isolated a TGase-producing strain S. hygroscopicus WSH03-13 and cloned the TGase ORF with a flanking sequence $[10,20]$. According to the sequence 


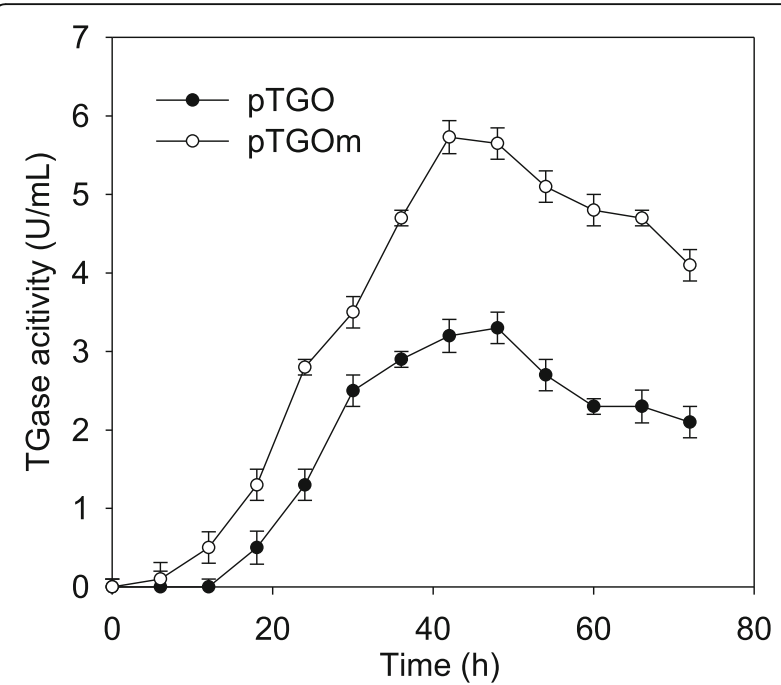

Fig. 4 The effect of codon optimization on the expression of TGase in S. lividans. The plasmid PTGO encoded TGase ORF with the optimized promoter (see Fig. 2b). The plasmid pTGOm encoded the same promoter as PTGO, and the TGase ORF was optimized according to the codon preference of S. lividans (see Fig. 3). The recombinant S. lividans TK24 that expressed pTGO or pTGOm was inoculated into $30 \mathrm{~mL}$ of medium (which contained $50 \mu \mathrm{g} / \mathrm{mL}$ apramycine) and cultured at $30^{\circ} \mathrm{C}$ and $200 \mathrm{rpm}$ for 3 days

analysis, the upstream sequence of the ORF contained a putative promoter [10]. However, the efficiency and the exact site of this endogenous promoter were not clear. Expression of the TGase ORF with the upstream sequence obtained extracellular TGase activity in S. lividans, confirming the existence of the endogenous promoter (Fig. 1). Based on deletion analysis, the complete sequence of the TGase endogenous promoter is restricted to the region from -693 to -48 , and a negative element ( -198 to -148$)$ was identified (Fig. 2). Finally, the TGase production in S. lividans was increased by $81.3 \%$ through the deletion of this element (Fig. 2b). Further investigation should be focused on the action mode of the negative element, which may serve to understand the physiological function of the TGase in Streptomyces.

Codon optimization was also used to improve the TGase expression in S. lividans. There is evidence of improved expression in the host strain when certain rare codons are replaced with preferred codons [24-26]. This phenomenon is thought to be related to the relative levels of the intracellular pool of charged transfer RNA molecules, which are low for rare codons and high for abundant codons [27]. As indicated by our previous study [10], S. hygroscopicus TGase ORF contains a rare codon TTA (leucine, codon usage $0.2 \%$ ) in Streptomyces [28]. Thus, it could prevent TGase expression because of the low level of transfer RNA molecules. In this study, the gene sequence optimization of TGase ORF according to the codon bias of Streptomyces resulted in $73.6 \%$ enhanced TGase production in S. lividans. To be noted, the codon optimization reduced the fermentation period for the highest TGase activity by $6 \mathrm{~h}$ (Fig. 4). After the sequence optimization, TTA that encoded Leu in the TGase ORF was mutated to the Streptomyces preferred codon CTC (Fig. 3). Because bldA, which encodes tRNA(Leu) (UUA), has been reported to be expressed only during the late stage of growth [28], the replacement of TTA by the Streptomyces preferred codon could account for the reduced fermentation period of the cells that expressed optimized TGase gene.

To further increase the production level of recombinant TGase at a large scale, the optimization medium and culture conditions will be performed in fermentors $[29,30]$.

\section{Conclusions}

In conclusion, we constructed an active TGaseproducing strain with a high yield and productivity, which could be a good candidate strain for industrial production of this enzyme. Moreover, the optimized TGase promoter and site-directed of rare codon TTA may also useful for improving other protein expression in S. lividans.

\section{Methods}

Bacterial strains, plasmids, and culture conditions

S. hygroscopicus WSH03-13 that produces TGase was stored in our lab [10]. E. coli JM109 was used for gene cloning. S. lividans TK24 (Str-6, tipAp induced, SLP2-, SLP3-) and pIJ86 (Streptomyces complementation plasmid; oriColE1 SCP2* aac(3)IV ermE* p) were used as the expression host and plasmid, respectively. Streptomyces cultures were grown on R2YE agar [31] or in liquid that contained glycerol $20 \mathrm{~g} / \mathrm{L}$, peptone $20 \mathrm{~g} / \mathrm{L}$, yeast extract $5 \mathrm{~g} / \mathrm{L}, \mathrm{MgSO}_{4} 2 \mathrm{~g} / \mathrm{L}, \mathrm{K}_{2} \mathrm{HPO}_{4} 2 \mathrm{~g} / \mathrm{L}, \mathrm{KH}_{2} \mathrm{PO}_{4} 2 \mathrm{~g} / \mathrm{L}$, and $\mathrm{CaCl}_{2} 1 \mathrm{~g} / \mathrm{L}$. A loop of fresh spore suspension of $S$. hygroscopicus WSH03-13 or S. lividans TK24 was inoculated into $30 \mathrm{~mL}$ of medium and cultured at $30{ }^{\circ} \mathrm{C}$ and $200 \mathrm{rpm}$ for 2-3 days. E. coli JM109 was grown in Luria-Bertani medium at $37^{\circ} \mathrm{C}$.

\section{Construction of plasmids that express TGase with their endogenous promoter}

To obtain a plasmid that expresses TGase with its endogenous promoter, a 2.6-kb DNA fragment $\operatorname{tg} 1)$ that contained the TGase gene with a flanking sequence from S. hygroscopicus WSH03-13 was amplified by PCR using the primer pairs TGUF/TGDR (Table 1), and the fragment was then inserted into the Kpn I-Bgl II sites of pIJ86, which resulted in the plasmids pIJ86/tg1 (Fig. 1a). 
Table 1 Primers used in this study

\begin{tabular}{ll}
\hline Primer & Sequence $\left(5^{\prime}-\right.$ 3') $^{\prime}$ \\
\hline TGUF & CGGGGTACCCGTAGCGGGTGGCGAAGAT \\
TGDR & GGAAGATCTCACGAGGACACCGAACGACTG \\
TG100F & TCGAGCTCGGTACCCACCCCGCTGAATGGGACTCTTCGT \\
TG200F & TTCGAGCTCGGTACCCCAGGAGCAGGGGACGCTGC \\
TG300F & TTCGAGCTCGGTACCGACGTGCCGGGGAGTGGCGC \\
TG400F & TTCGAGCTCGGTACCCTCTCCCTGCGGTCGCCGTGACAG \\
TGQ1R & ACATGCATGCGACCTCAGCCGCGCTGTCCTGGGTC \\
TGQ2R & ACATGCATGCCCGCCACGAGGCGGAAGGAGATGC \\
TGQ3R & ACATGCATGCGCGTGGCCGTCGCCGGTCATGACCTGGTG \\
TGQ4R & ACATGCATGCGGCGGCACCGGTGCCTCGCTACATC \\
TGQ5R & ACATGCATGCGGGCCCGTCCGGGGGCCAGG \\
TGQ6R & ACATGCATGCGGGAGTCATGAAGTCGGTGTC \\
TGQ7R & ACATGCATGCACAGCGGCGGTCGCGGGGGACGG \\
TGQ8R & ACATGCATGCGCCTCGCCGCGAACCGCACGCCAGG \\
TGQ9R & ACATGCATGCGGCAGGTCGGGAGCGCCTGTC
\end{tabular}

\section{Construction of plasmids that express TGase with partially deleted endogenous promoters}

To partially delete the $5^{\prime}$-end of the promoter region, each gene fragment of $\operatorname{tg} 1$ with a 5 '-end deletion at the promoter region (Fig. 2a) was amplified from pIJ86/tg1 by PCR using a specific forward primer and a constant reverse primer (TGDR) (Table 1). For the deletion of the first $100 \mathrm{bp}$ nucleotides at the $5^{\prime}$-end of the promoter region, TG100F (Table 1) was used as a forward primer. For further deletions at the $5^{\prime}$-end of the promoter region, TG200F, TG300F, and TG400F were in turn used as the forward primer (Table 1). The resulting PCR products were inserted into the $K p n$ I- $B g l$ II sites of pIJ86 to produce pTGU2, pTGU3, pTGU4, and pTGU5, respectively (Fig. $2 \mathrm{a}$ ).

To partially delete the 3 '-end of the promoter region, the gene fragment that contained the complete open reading frame (ORF) of TGase and the fragments that encoded the promoter region with 3 '-end deletions were amplified from pIJ86/tg1 by PCR, separately (Fig. 2b). Each gene fragment that encoded the promoter region with the 3 '-end deletion was obtained by using a constant forward primer (TGUF) and a specific reverse primer (Table 1). For the deletion of the first $50 \mathrm{bp} \mathrm{nu-}$ cleotides at the 3 '-end of the promoter region (Fig. 2b), TGQ1R (Table 1) was used as a reverse primer. For further 3'-end deletion of the promoter, TGQ2R, TGQ3R, TGQ4R, TGQ5R, TGQ6R, TGQ7R, TGQ8R, and TGQ9R were in turn used as the forward primer (Table 1). The resulting PCR products were inserted into the Kpn I-Sph I sites of pIJ86/tg1 to produce pTGD2, pTGD3, pTGD4, pTGD5, pTGD6, pTGD7, pTGD8, pTGD9, and pTGD10, respectively (Fig. 2b).
The negative element (-197 to -149$)$ was removed from the TGase promoter (-693 and -48$)$ by chemical synthesis, and the resulting gene fragment was cloned into the Kpn I-Sph I sites of pIJ86/tg1 to produce pTGO (Fig. 2b).

\section{Codon optimization of the TGase gene in S. lividans}

According to the codon preference of S. lividans, the $S$. hygroscopicus TGase ORF was optimized and synthesized by Genscript (Nanjing, China). The codon-optimized TGase ORF with an intact upstream (-48 to -1$)$ and downstream (1258 to 1715) was then cloned into the Sph I- $B g l$ II sites of pTGO, which yielded pTGOm.

\section{Expression of the TGase gene in S. lividans}

Molecular methods for Stretomyces were used as described by Hopwood et al. [31]. Plasmids that expressed TGase with an endogenous promoter or its partially deleted versions were transformed into S. lividans TK24. The S. lividans transformants were selected on a plate that contained $50 \mu \mathrm{g} / \mathrm{mL}$ apramycine. When the transformants were grown in liquid medium, $50 \mu \mathrm{g} / \mathrm{mL}$ apramycine were added. The recombinant S. lividans TK24 were inoculated into $30 \mathrm{~mL}$ of medium and cultured at $30{ }^{\circ} \mathrm{C}$ and $200 \mathrm{rpm}$ for $2-3$ days.

\section{Assay of TGase activity}

TGase activity was measured using a colorimetric procedure in which $\mathrm{N}$ - $\alpha$-carbobenzoxyl-glutaminyl-glycine (N-CBZ-Gln-Gly) (Sigma, Shanghai, China) was used as the substrate [8]. Forty microliters of substrate solution (30 mmol/L N-CBZ-Gln-Gly, $100 \mathrm{mmol} / \mathrm{L}$ hydroxylamine, $10 \mathrm{mmol} / \mathrm{L}$ glutathione, $200 \mathrm{mmol} / \mathrm{L}$ Tris- $\mathrm{HCl}$ buffer, pH6) was added to $100 \mu \mathrm{L}$ of TGase solution to initiate the enzymatic reaction. After $10 \mathrm{~min}$, the reaction was stopped by the addition of a $40-\mu \mathrm{L}$ terminator $(1 \mathrm{~mol} / \mathrm{L} \mathrm{HCl}, 4 \%(\mathrm{v} / \mathrm{v})$ trichloroacetic acid, $2 \%(\mathrm{~m} / \mathrm{v})$ $\mathrm{FeCl}_{3} \cdot 6 \mathrm{H}_{2} \mathrm{O}$ ), and the reaction solution was subjected to spectrophotometry analysis at $525 \mathrm{~nm}$. A calibration curve was obtained using L-glutamic acid $\gamma$-monohydroxamate (Sigma, Shanghai, China). One unit of TGase was defined as that required to generate $1 \mu \mathrm{mol}$ of L-glutamic acid $\gamma$ monohydroxamate per min at $37^{\circ} \mathrm{C}$.

\section{Protein analysis}

SDS-PAGE was performed on a $12 \%$ running gel, and the resolved proteins were visualized by staining with Coomassie Brilliant Blue R250. Protein concentrations were measured using the Bradford method, with bovine serum albumin as the standard.

\section{Abbreviations}

N-CBZ-Gln-Gly: N-a-carbobenzoxyl-glutaminyl-glycine; ORF: complete open reading frame; TGase: transglutaminase 


\section{Acknowledgments}

We would like to thank Professor Nam Sun Wang for critically reading and providing suggestions for the manuscript.

\section{Funding}

This work was financially supported by the National Natural Science Foundation of China (No. 31401638), the National High Technology Research and Development Program of China (No. 2015AA021003), the Key Technologies R \& D Program of Jiangsu Province (No. BE2016629), and the Natural Science Foundation of Jiangsu Province (No. BK20130132).

\section{Availability of data and materials}

The Nucleic acids sequence supporting the conclusions of this article is available in the GenBank (National Center for Biotechnology Information) [http://www.ncbi.nlm.nih.gov/genbank]. The TGase gene with a flanking sequence from Streptomyces hygroscopicus WSH03-13 Accession: HM231108.

\section{Authors' contributions}

SL conducted the molecular genetic studies and drafted the manuscript. MW participated in the design of the study. GD and JC conceived the study and participated in its design and coordination and helped to draft the manuscript. All of the authors read and approved the final manuscript.

\section{Competing interests}

All authors declare that they have no competing interests.

\section{Consent for publication}

Not applicable.

\section{Ethics approval and consent to participate}

Not applicable.

\section{Author details \\ ${ }^{1}$ Key Laboratory of Industrial Biotechnology, Ministry of Education, School of Biotechnology, Jiangnan University, Wuxi, China. ${ }^{2}$ National Engineering Laboratory for Cereal Fermentation Technology, Jiangnan University, Wuxi, China. ${ }^{3}$ Key Laboratory of Carbohydrate Chemistry and Biotechnology, Ministry of Education, School of Biotechnology, Jiangnan University, Wuxi, China. ${ }^{4}$ School of Food Science and Technology, Jiangnan University, Wuxi 214122, China.}

Received: 16 September 2015 Accepted: 13 October 2016 Published online: 28 October 2016

\section{References}

1. Yokoyama K, Nio N, Kikuchi Y. Properties and applications of microbial transglutaminase. Appl Microbiol Biotechnol. 2004;64(4):447-54

2. Martins IM, Matos M, Costa R, Silva F, Pascoal A, Estevinho LM, Choupina AB. Transglutaminases: recent achievements and new sources. Appl Microbiol Biotechnol. 2014;98(16):6957-64.

3. Serafini-Fracassini D, Del Duca S. Transglutaminases: widespread crosslinking enzymes in plants. Ann Bot. 2008;102(2):145-52.

4. Nemes Z, Marekov LN, Fesus L, Steinert PM. A novel function for transglutaminase 1: attachment of long-chain omega-hydroxyceramides to involucrin by ester bond formation. Proc Natl Acad Sci U S A. 1999;96(15): 8402-7.

5. Zhang D, Zhu Y, Chen J. Microbial transglutaminase production: understanding the mechanism. Biotechnol Genet Eng Rev. 2010;26:205-22.

6. Yang MT, Chang CH, Wang JM, Wu TK, Wang YK, Chang CY, Li TT. Crystal structure and inhibition studies of transglutaminase from Streptomyces mobaraense. J Biol Chem. 2011;286(9):7301-7.

7. Kashiwagi T, Yokoyama K, Ishikawa K, Ono K, Ejima D, Matsui H, Suzuki E. Crystal structure of microbial transglutaminase from Streptoverticillium mobaraense. J Biol Chem. 2002;277(46):44252-60.

8. Liu S, Zhang DX, Wang M, Cui WJ, Chen KK, Du GC, Chen J, Zhou ZM. The order of expression is a key factor in the production of active transglutaminase in Escherichia coli by co-expression with its pro-peptide. Microb Cell Fact. 2011;10:112

9. Yurimoto H, Yamane M, Kikuchi Y, Matsui H, Kato N, Sakai Y. The propeptide of Streptomyces mobaraensis transglutaminase functions in cis and in trans to mediate efficient secretion of active enzyme from methylotropic yeasts. Biosci Biotechnol Biochem. 2004;68(10):2058-69.

10. Liu S, Zhang DX, Wang M, Cui WJ, Chen KK, Liu Y, Du GC, Chen J, Zhou ZM. The pro-region of Streptomyces hygroscopicus transglutaminase affects its secretion by Escherichia coli. FEMS Microbiol Lett. 2011;324(2):98-105.

11. Yu YJ, Wu SC, Chan HH, Chen YC, Chen ZY, Yang MT. Overproduction of soluble recombinant transglutaminase from Streptomyces netropsis in Escherichia coli. Appl Microbiol Biotechnol. 2008;81(3):523-32.

12. Kikuchi $Y$, Date M, Yokoyama K, Umezawa Y, Matsui H. Secretion of active-form Streptoverticillium mobaraense transglutaminase by Corynebacterium glutamicum: Processing of the pro-transglutaminase by a cosecreted subtilisin-like protease from Streptomyces albogriseolus. Appl Environ Microbiol. 2003;69(1):358-66.

13. Washizu K, Ando K, Koikeda S, Hirose S, Matsuura A, Takagi H, Motoki M, Takeuchi K. Molecular-cloning of the gene for microbial transglutaminase from Streptoverticillium and its expression in Streptomyces-lividans. Biosci Biotechnol Biochem. 1994;58(1):82-7.

14. Lin YS, Chao ML, Liu CH, Chu WS. Cloning and expression of the transglutaminase gene from Streptoverticillium ladakanum in Streptomyces lividans. Process Biochem. 2004:39(5):591-8.

15. Lin YS, Chao ML, Liu CH, Tseng M, Chu WS. Cloning of the gene coding for transglutaminase from Streptomyces platensis and its expression in Streptomyces lividans. Process Biochem. 2006;41(3):519-24.

16. Ali N, Herron PR, Evans MC, Dyson PJ. Osmotic regulation of the Streptomyces lividans thiostrepton-inducible promoter, ptipA. Microbiol-Sgm. 2002;148:381-90.

17. Liu X, Yang X, Xie F, Qian S. Cloning of transglutaminase gene from Streptomyces fradiae and its enhanced expression in the original strain. Biotechnol Lett. 2006:28:1319-25.

18. Ueda Y, Taguchi S, Nishiyama K, Kumagai I, Miura K. Effect of a rare leucine codon, TTA, on expression of a foreign gene in Streptomyces lividans. Biochim Biophys Acta. 1993;1172(3):262-6.

19. Chen K, Zhang D, Liu S, Wang NS, Wang M, Du G, Chen J. Improvement of transglutaminase production by extending differentiation phase of Streptomyces hygroscopicus: mechanism and application. Appl Microbiol Biotechnol. 2013;97(17):7711-9.

20. Cui L, Du GC, Zhang DX, Liu H, Chen J. Purification and characterization of transglutaminase from a newly isolated Streptomyces hygroscopicus. Food Chem. 2007;105(2):612-8.

21. Noda S, Miyazaki T, Tanaka T, Chiaki O, Kondo A. High-level production of mature active-form Streptomyces mobaraensis transglutaminase via protransglutaminase processing using Streptomyces lividans as a host. Biochem Eng J. 2013;74:76-80.

22. Marx CK, Hertel TC, Pietzsch M. Purification and activation of a recombinant histidine-tagged pro-transglutaminase after soluble expression in Escherichio coli and partial characterization of the active enzyme. Enzyme Microb Technol. 2008;42(7):568-75

23. Kim DW, Kang SG, Kim IS, Lee BK, Rho YT, Lee KJ. Proteases and protease inhibitors produced in streptomycetes and their roles in morphological differentiation.J Microbiol Biotechn. 2006:16(1):5-14

24. McVey JH, Ward NJ, Buckley SMK, Waddington SN, VandenDriessche T, Chuah MKL, Nathwani AC, McIntosh J, Tuddenham EGD, Kinnon C, et al. Codon optimization of human factor VIII cDNAs leads to high-level expression. Blood. 2011:117(3):798-807.

25. Fang BS, Li W, Ng IS, Yu JC, Zhang GY. Codon optimization of 1,3 propanediol oxidoreductase expression in Escherichia coli and enzymatic properties. Electron J Biotechnol. 2011;14(4):7.

26. Hou Y, Wang H, Wang QL, Zhang FF, Huang YH, Ji YL. Protein expression and purification of human Zbtb7A in Pichia pastoris via gene codon optimization and synthesis. Protein Expr Purif. 2008;60(2):97-102.

27. Binnie C, Cossar JD, Stewart DI. Heterologous biopharmaceutical protein expression in Streptomyces. Trends Biotechnol. 1997;15(8):315-20.

28. Liu L, Yang H, Shin HD, Li J, Du G, Chen J. Recent advances in recombinant protein expression by Corynebacterium, Brevibacterium, and Streptomyces: from transcription and translation regulation to secretion pathway selection. Appl Microbiol Biotechnol. 2013;97(22): 9597-608.

29. Lin SJ, Hsieh YF, Lai LA, Chao ML, Chu WS. Characterization and large-scale production of recombinant Streptoverticillium platensis transglutaminase. J Ind Microbiol Biotechnol. 2008;35(9):981-90. 
30. Noda S, Miyazaki T, Tanaka T, Ogino C, Kondo A. Production of Streptoverticillium cinnamoneum transglutaminase and cinnamic acid by recombinant Streptomyces lividans cultured on biomass-derived carbon sources. Bioresour Technol. 2012:104:648-51.

31. Bibb MJ TK, MJ B, KF C, DA H. Practical Streptomyces Genetics. 2nd ed. Norwich: The John Innes Foundation; 2000.

Submit your next manuscript to BioMed Central and we will help you at every step:

- We accept pre-submission inquiries

- Our selector tool helps you to find the most relevant journal

- We provide round the clock customer support

- Convenient online submission

- Thorough peer review

- Inclusion in PubMed and all major indexing services

- Maximum visibility for your research

Submit your manuscript at www.biomedcentral.com/submit 